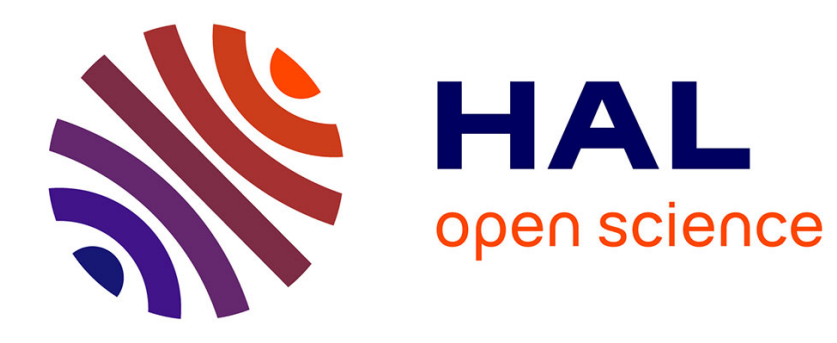

\title{
L'accentuation des noms des villes en anglais Bruno Delaroche
}

\section{To cite this version:}

Bruno Delaroche. L'accentuation des noms des villes en anglais. Nouvelle Revue d'Onomastique, 2010, 52 (1), pp.319-329. 10.3406/onoma.2010.1550 . hal-02471941

\section{HAL Id: hal-02471941 \\ https://hal.science/hal-02471941}

Submitted on 9 Feb 2020

HAL is a multi-disciplinary open access archive for the deposit and dissemination of scientific research documents, whether they are published or not. The documents may come from teaching and research institutions in France or abroad, or from public or private research centers.
L'archive ouverte pluridisciplinaire HAL, est destinée au dépôt et à la diffusion de documents scientifiques de niveau recherche, publiés ou non, émanant des établissements d'enseignement et de recherche français ou étrangers, des laboratoires publics ou privés. 


\section{L'accentuation des noms des villes en anglais}

Bruno Delaroche

Citer ce document / Cite this document :

Delaroche Bruno. L'accentuation des noms des villes en anglais. In: Nouvelle revue d'onomastique, n52, 2010. pp. 319-329;

doi : https://doi.org/10.3406/onoma.2010.1550

https://www.persee.fr/doc/onoma_0755-7752_2010_num_52_1_1550

Fichier pdf généré le 04/04/2018 


\author{
Bruno Delaroche \\ Université Rennes 2
}

\title{
L'accentuation des noms de ville en anglais
}

\section{Introduction}

Le but de la présente étude est de tenter de voir s'il existe des constantes au niveau de l'accentuation des toponymes en anglais permettant de dégager des régularités en ce qui concerne la place de l'accent tonique dans un toponyme composé présentant au minimum un terme immédiatement compréhensible, sans avoir à se reporter à un dictionnaire. Le cas des toponymes composés « transparents ", ou " semi-transparents ", nous a semblé intéressant car, contrairement aux toponymes " opaques », ils intègrent des éléments, tels que des noms ou des adjectifs, porteurs de sens et donc plus ou moins facilement identifiables sans faire appel à l'étymologie.

En analysant des noms de ville ou de quartiers de ville, principalement choisis en Angleterre avec quelques exemples pris aux États-Unis, en Irlande, au Pays de Galles et en Écosse, nous verrons si l'accentuation de ces toponymes obéit aux mêmes règles que celles régissant les noms communs composés en anglais, ou si les caractéristiques fonctionnelles jouent un rôle dans le positionnement de l'accent. Les toponymes autres que ceux désignant des villes, ou des quartiers, ne seront pas traités ici car l'utilisation d'un terme générique, tel que les mots Sea, Ocean, Mount, Lake, etc., accompagnant le toponyme, peut avoir une incidence sur la place de l'accent.

En anglais l'accent tonique d'un mot est placé par défaut sur la première syllabe. Ce qui peut entraîner un déplacement de cet accent, c'est essentiellement la présence d'un préfixe ou d'un suffixe, ou lorsqu'on a affaire à un mot composé.

La majorité des noms de ville en anglais suit ce principe, qu'il s'agisse de toponymes simples ou complexes, transparents, semi-transparents ou opaques :

'London, 'Bristol, 'Edinburgh, 'Derby, 'Coventry, 'Liverpool, 'Winchester,

'Peterborough, 'Washingborough, 'Margate, 'Shrewsbury, 'Sheffield,

'Gainsborough, 'Chippenham, 'Chesterfield, 'Chesterton, 'Little,hampton,

'Littleborough ou 'Litlle,borough ...

Certains toponymes, en revanche, sont accentués sur d'autres syllabes que la première. Carlisle par exemple est accentué sur la deuxième syllabe, mais localement sur la première.

Dans le cas de Wolverhampton, l'accent est placé indifféremment sur la première ou la troisième syllabe. Piccadilly comporte un accent principal sur la troisième syllabe et un accent secondaire sur la première syllabe. Aberdeen est accentué sur la dernière syllabe, mais Kirkcaldy et Tipperary sont accentués sur l'avant dernière syllabe. 
Notre étude portera majoritairement sur des noms de villes anglaises car l'accentuation des noms de villes américaines ne semble pas aussi systématique. Si un certain nombre d'entre elles portent un accent tonique sur la première syllabe, dans beaucoup de cas la place de cet accent varie énormément :

'Washington, 'Jackson, ville, 'Harrisburg, 'Providence, 'Charleston, 'Charlottesville,

'Houston, 'Portland, 'Dallas, 'Nashville, 'Detroit, 'Cleveland, 'Baltimore, 'Norfolk,

'Pittsburg, 'Middles, borough/'Middles, boro (accent sur la première syllabe).

Mais d'autres toponymes sont accentués sur la deuxième ou troisième syllabe, avec parfois un accent secondaire placé sur la première syllabe, comme dans les exemples suivants :

A'tlanta, Or'lando, Chi'cago, Se'attle, Mil'waukee, Mi'ami, Brook'haven, Co'lumbus, Sa'vannah, Gil'lette, To'peka, ,Monti'cello, Cincin'nati, ,India'napolis, ,Sacra'mento, , Minne'apolis, ,Inde'pendence, ,Monter'rey, ...

En ce qui concerne les mots composés, l'accentuation obéit à plusieurs règles que nous rappellerons le moment venu. Les toponymes composés, qui feront l'objet de cette présentation, peuvent être formés en anglais selon plusieurs schémas :

Adjectif + nom, comme dans Whitefield ou Little Rock.

Nom + nom, comme dans Birkenhead ou Moot Hill.

Toponymes incluant un génitif, comme dans Kingsbridge, ou Bishop's Cleeve.

Toponymes composés de plus de deux constituants, comme dans Normanton le Heath ou Standford in the Vale.

Nous analyserons donc successivement ces différents modèles en établissant pour la plupart une différenciation entre toponymes soudés et non soudés et en faisant référence à leur structure fonctionnelle en reprenant la terminologie utilisée par Jonas Löfström et Betina Schnabel dans leur article de ce volume de la Nouvelle Revue d'Onomastique «Comment analyser et comparer les toponymes de différentes langues dans une perspective synchronique $»$.

\section{Adjectif + nom}

En anglais, dans les noms composés formés d'un adjectif et d'un nom, c'est le déterminant, c'est-à-dire le premier terme, qui porte l'accent principal, le déterminé (le second terme) perdant son accent ou voyant son accent s'estomper en accent secondaire, alors que dans les combinaisons adjectif + nom non-soudés, c'est en général le déterminé qui est accentué.

Ex : a 'greenhouse = une serre ; a green 'house = une maison verte;

a 'blueberry = une myrtille; a blue 'berry = une baie bleue

Les noms de ville composés d'un adjectif et d'un nom en anglais peuvent être classés dans plusieurs catégories en fonction du type d'adjectif présent dans le toponyme - adjectif de couleur, adjectif descriptif, points cardinaux. 


\section{L'accentuation des noms de ville en anglais}

\subsection{Adjectifs de couleur}

\subsubsection{Toponymes soudés}

Ce type de toponymes suit en général la règle que nous venons de rappeler. Ils sont donc accentués en général sur la première syllabe du premier terme :

'Whitechapel, 'Whitehaven, 'Whitefield, 'Whitehall, Whitehouse (Texas), 'Blackburn, 'Blackpool, 'Blackford, 'Greenfield, 'Greenwood, 'Redfield, 'Whitefriars (nom évoquant les White friars, moines carmélites et leurs habits blancs).

Exception : Black'friars (du nom des frères prêcheurs dominicains portant des habits de couleur noire). Il est curieux de constater que malgré la similitude de construction entre Whitefriars et Blackfriars, l'un est accentué sur la première syllabe et l'autre sur la seconde.

\subsubsection{Toponymes non-soudés}

Contrairement à ce que nous avons vu avec les exemples du type blue berry et green house, structure dans laquelle l'accent tonique est placé sur le déterminé, dans les exemples qui suivent l'accent tonique porte sur le premier élément, peut-être parce qu'il s'agit de noms propres apparentés à des concepts comme blueberry, qui désigne un fruit particulier de couleur bleutée et non pas un terme générique applicable à tous les types de baies bleues.

'White House (ville du Tennessee et Résidence du Président des États-Unis)

'White Hall (villes de l'Arkansas et de l'Illinois)

Dans tous les toponymes mentionnés ci-dessus dans les sous-chapitres 1.1 .1 et 1.1.2, le schéma fonctionnel est du type $\mathrm{As}+\mathrm{Ag}$.

\subsection{Adjectifs descriptifs}

Un grand nombre de noms de ville en anglais comportent des adjectifs descriptifs comme « new », « old », " long », " broad », " great » " high », .. (neuf, vieux, long, grand [par la taille ou au sens figuré], haut...), ou faisant référence à une caractéristique géologique ou climatique du lieu considéré, comme stony (pierreux), sandy (sablonneux), fenny (marécageux), dry ( $\mathrm{sec}$ ), cold (froid) etc., comme le souligne Kenneth CAMERON dans son ouvrage sur les toponymes britanniques English Place Names, p. 104.

\subsubsection{Toponymes soudés}

Ici encore le principe des noms composés que nous avons vu avec le mot "greenhouse » prédomine, ces toponymes étant majoritairement accentués sur la première syllabe du mot : 
'Newhaven, 'Newmarket, 'Newport, 'Newbury, 'Oldbury, 'Highgate, 'Highwood, 'Highbridge, 'Greathead, 'Greatheart, 'Littlehampton, 'Longfield, 'Longview, 'Longford, 'Longwood, 'Broadview, 'Broadfield, 'Broadway, 'Dryburgh ...

Le schéma fonctionnel de ces toponymes est du type As + Ag.

\subsubsection{Toponymes non-soudés}

Comme pour les adjectifs de couleurs, dans les noms de ville incluant un adjectif descriptif composé de deux termes non-soudés l'accent porte sur le second terme, le premier terme ayant vraisemblablement à l'origine toujours sa valeur d'adjectif. Ce type de toponymes est essentiellement composé selon le schéma $\mathrm{As}+\mathrm{Pg}$ :

Long 'Eaton, New 'Orleans, New 'Romney, New 'York, Great 'Yarmouth, High

'Wycombe, Old 'Fletton, Stony 'Stratford, Stony 'Middleton, Dry 'Drayton, Dry

'Doddington,

En revanche 'Little Rock (As $+\mathrm{Ag})$, est accentué sur le premier terme. Peut-être parce que le second terme est un nom commun, alors que dans les exemples précédents il s'agissait de noms propres.

\subsection{Points cardinaux}

\subsubsection{Toponymes soudés}

Ce type de toponymes fonctionne comme ceux composés avec un adjectif descriptif présentés au-dessus. Les toponymes soudés portent donc majoritairement un accent tonique sur la première syllabe du terme :

'Northbridge, 'Northbrook, 'Northfield, 'Southgate, 'Southport, 'Southbridge,

'Southwold, 'Southsea, 'Eastwood, 'Westfield, (As + Ag), 'Westmorland,

'Eastbourne, (As + Pg).

Exceptions: Westminster pour lequel l'accent peut porter soit sur la première syllabe, soit sur la deuxième. Par ailleurs, South'end, South'ampton, North'ampton sont accentués sur la deuxième syllabe.

\subsubsection{Toponymes non-soudés}

Pour ces toponymes, l'accent porte sur la première syllabe du second terme, comme pour la majorité des noms communs construits avec un adjectif qui décrit la nature du nom. Comme pour les composés soudés, le schéma fonctionnel peut être soit $\mathrm{As}+\mathrm{Ag}$, soit As + Pg :

South 'Haven, South 'Bend. South 'Bay, South 'Shields, (As + Ag).

South 'Berwick, South'Boston, South 'Barwon, East 'Lyme, West 'Caldwell, West 'Brookfield (As + Pg). 


\section{L'accentuation des noms de ville en anglais}

\section{Nom + nom}

En anglais l'accentuation des noms composés constitués de deux noms dépend de plusieurs facteurs, mais la plupart du temps l'accent porte sur le premier terme, que les mots soient soudés ou non soudés. C'est le cas notamment lorsqu'il y a présence d'un mot à valeur de suffixe, (comme -man, -woman, -person, -berry, -day, -folk, -house, time, -ware, -land ['night-time (la nuit), 'chairman (président), 'kinfolk (ou 'kinsfolk, membre de la famille), 'glassware (verrerie), 'glasshouse/'greenhouse (serre)...]). Cette présence d'un suffixe se retrouve également en anglais (ainsi que dans d'autres langues) dans certains toponymes, comme le rappelle l'auteur de l'ouvrage Theory and Typology of Proper Names, (Willy VAN LANGENDONK, 2007, page 205). L'accent tonique est également placé sur le premier terme dans les noms dont le second élément désigne un agent formé d'un verbe accompagné du suffixe «er», comme 'bookseller (libraire), ou un agent formé d'un verbe et d'un suffixe en "ing ", comme 'fox-hunting " (chasse au renard), ou dans les noms composés d'un verbe combiné à un complément, comme 'passport, les nom composés qui comportent un mot en « in »g comme premier élément, comme 'rocking-chair, ou encore les noms composés dont le premier élément caractérise le second, comme 'skinhead.

Les toponymes faisant partie de cette catégorie peuvent être composés de noms communs ou d'un terme générique accompagné d'un proprial, le nom commun pouvant se trouver en première ou seconde position dans le nom de lieu ainsi constitué. Parmi les termes génériques les plus courants dans ce genre de formation, on trouve des mots désignant des constructions, comme bridge (pont), church (église), minster (monastère), castle (château), des noms faisant référence à la topographie, comme wood (forêt), field (champ), dale (vallée), marsh ou fen (marais), hill (colline), cliff (falaise), mouth (estuaire), ou encore des termes comme port ou harbour (port).Les termes désignant des fonctions (politiques ou ecclésiastiques) sont également nombreux dans les toponymes transparents ou semi-transparents: king, ou la forme latine regis (roi), queen (reine), knight (chevalier), abbot (abbé), priest (prêtre), bishop (évêque), ainsi que les toponymes associés à des noms de saints.

\subsection{Toponymes soudés}

L'accent porte dans la majorité des cas sur la première syllabe du premier terme, quel que soit le schéma fonctionnel des toponymes considérés :

'Bridgeport, 'Bridgewater (ville des États-Unis) et 'Bridgwater (ville d'Angleterre),

'Bridge-view, 'Bridgetown, 'Bridgeburg, 'Fordingbridge, 'Ivybridge, 'Stone, haven,

'Maiden, head, 'Maidstone/'Maid,stone (As + Ag).

En revanche Bridg'end est accentué sur le second constituant, peut-être parce que le point de vue n'est pas le même que dans les autres exemples comportant le mot bridge, ou en raison de l'élision du e de bridge. Quant à Birkenhead, le dictionnaire américain Merriam-Webster's Geographical Dictionary indique deux accentuations possibles : un accent principal sur la première syllabe du mot et un accent secondaire sur le second 
constituant ou l'inverse, alors que l'English Pronouncing Dictionary de Daniel JONES place l'accent principal sur head et un accent secondaire sur la première syllabe du toponyme.

'Avondale, et 'Avonmouth constitués de Ps $+\mathrm{Ag}$, ainsi qu' Ashbourne et 'Ashburton $(\mathrm{As}+\mathrm{Pg})$ sont eux aussi accentués sur le premier terme.

\subsection{Toponymes non-soudés}

Comme dans le cas des noms de ville comportant des points cardinaux et constitués de deux composants non-soudés, les toponymes composés de deux noms (nom propre + nom commun ou nom commun + nom propre), sont en général accentués sur le second constituant :

Market 'Rasen, Market 'Deeping, Priest 'Weston, Ag + Pg

Astley 'Abbots, Bognor 'Regis, Lynn 'Regis, Lyme 'Regis, Ps + Ag.

Dans le cas de ces deux derniers exemples, nous pouvons faire remarquer que le Merriam-Webster's Geographical Dictionary indique deux accents (un sur la première syllabe de chacun des termes), alors que le JONES ne donne qu'un seul accent sur le second constituant.

Downham 'Market et Walton 'Heath, constitućs de $\mathrm{Pg}+\Lambda \mathrm{g}$, suivent le même principe de l'accent tonique placé sur le second élément qui rappelle ce qui se passe en anglais dans les composés comportant un adjectif et un nom comme dans short 'circuit (court-circuit), full 'moon (pleine lune), public 'bar, etc. Saint :

La même chose se produit pour les noms de ville en anglais comportant le mot

Saint 'Albans, Saint 'Andrews, Saint 'Paul, Saint 'Peter, Ag + Pg

\section{Toponymes incluant un génitif}

Les noms de ville incluant un génitif, qu'il soit soudé ou non, ne sont pas rares en anglais. Dans les noms communs composés, qui comportent un génitif qui n'est plus séparé, l'accentuation est régulièrement à gauche :

'craftsman (artisan), 'foolscap (papier ministre), 'kinsfolk (membres de la famille), 'statesman (homme d'État), etc.

\subsection{Toponymes soudés}

Comme les noms communs que nous venons de mentionner, les noms de ville (ou de quartiers de ville) en anglais qui adoptent cette graphie sont généralement accentués à gauche, et sont d'ailleurs souvent constitués de deux noms communs. On retrouve dans ces toponymes le même genre de termes génériques que ceux mentionnés plus haut dans le chapitre 2.

'Bishopsgate, 'Bishopston, 'Kingston, 'Queensbury, ,'Kingswood, 'Kingsborough, 'Knights-bridge, 'Abbotsford, As + Ag. 


\subsection{Toponymes non-soudés}

D'autres noms de ville, en revanche, ont conservé la forme séparée du génitif, avec parfois une double orthographe, le $\mathrm{S}$ marquant le génitif pouvant être collé au nom ou séparé par une apostrophe comme dans la graphie classique. La disparition de l'apostrophe tient peut-être à des raisons purement pratiques de facilité d'emploi à l'écrit, étant donné qu'à l'oral la différence d'orthographe n'est pas perceptible.

King's 'Norton/Kings 'Norton ("Le Norton du roi") Bishop's 'Norton/Bishops 'Norton ("le Norton de l'évêque"), King's 'Lynn, Bishop's 'Stortford, Kings 'Walden/Kings 'Walden, Abbot's 'Barton ("le Barton de l'abbé"), Abbot's 'Bromley, As + Pg.

King's'Cliffe/Kings 'Cliffe, As + Ag.

,Martha's'Vineyard, (accent secondaire sur la première syllabe du premier terme), Ps + Ag.

Comme dans les génitifs déterminatifs en anglais, ces toponymes sont accentués à droite, indépendamment de leur schéma fonctionnel.

\section{Toponymes de plus de deux constituants}

En plus des toponymes de forme courante formés par l'ajout de préposition comme under (sous), on/upon (sur), in (en/dans) ou by (près de), certains noms de villes en anglais présentent des constructions plus originales, soit la simple juxtaposition de plusieurs termes (trois ou quatre), soit des éléments reliés par le mot le (parmi le/les), juxta (forme latine de by), super ou atte/at (au/à la). Ce type de toponymes comprend souvent deux accents, un accent secondaire sur le premier terme et un accent principal sur le dernier :

,Stockton on the 'Forest, ,Stockton-on-'Tees, Heddon on the 'Wall, ,Preston upon the 'Weald (Preston [le village des prêtres] dans la lande), ,North Weald 'Basset, ,Harrrow on the 'Hill, ,Welton le 'Marsh (Welton des marais), ,Welton le 'Wold (Welton dans la lande), Newton in 'Mackerfield, ,Newton-le-'Willows (la ville neuve parmi les saules), ,Normanby-le-'Wold (Normanby dans la lande), ,Westonsuper-'Mare, ,Newcastle-,under-'Lyme,(deux accents secondaires), Clayton-le'Moors, ,Houghton-le-'Spring, ,Houghton-le-'Side, ,Havering-atte-'Bower (Havering à la résidence du roi), ,Langton-juxta- 'Partney, ,Newton Saint 'Loes.

On assiste en fait avec ces toponymes à un déplacement de l'accentuation de la gauche vers la droite. Si on prend le cas de Stockton, par exemple, l'accent tonique porte sur la première syllabe, alors que dans Stockton-on-Tees cet accent s'est transformé en accent secondaire et l'accent principal porte sur le dernier élément. Ce phénomène est courant avec les noms de ville ou de quartiers de ville lorsque le toponyme est suivi d'un terme qui peut désigner un lieu mais pas exclusivement :

Picca'dilly $\rightarrow$,Piccadilly 'Circus, 'Hampstead $\rightarrow$,Hampstead 'Heath,

,Hyde 'Park $\rightarrow$,Hyde, Park 'Corner, 'Hampton $\rightarrow$, Hampton 'Court

'Chester $\rightarrow$,Chester-le- 'Street, 'Canterbury $\rightarrow$, Canterbury 'Tales. 
'New,castle $\rightarrow$,Newcastle upon 'Tyne, New 'York $\rightarrow$, New York 'City, 'Washington $\rightarrow$,Washington,$D$ 'C, 'Madison $\rightarrow$, Madison 'Avenue

\section{Tableaux récapitulatifs}

Le tableau suivant met en évidence les régularités que nous avons pu observer en ce qui concerne la place de l'accent tonique dans les toponymes soudés et non-soudés, classés en fonction des termes qui les composent (adjectif, nom, etc.). Les chiffres 1 et 2 indiquent quel constituant est accentué dans le toponyme.

5.1. Accentuation lexicale du toponyme par rapport au nombre de constituants (soudé et non-soudé comparé)

\begin{tabular}{|c|c|c|}
\hline \multirow{2}{*}{ Nom } & \multicolumn{2}{|c|}{ Constituant accentué } \\
\hline & Toponyme soudé & Toponyme non-soudé \\
\hline Whitefield & 1 & \\
\hline Whitehaven & 1 & \\
\hline Greenwood & 1 & \\
\hline Blackpool & 1 & \\
\hline Little Rock & & 1 \\
\hline White House & & 1 \\
\hline White Hall & & 1 \\
\hline Newhaven & 1 & \\
\hline Longview & 1 & \\
\hline Highbridge & 1 & \\
\hline Littlehampton & 1 & \\
\hline Littleborough & 1 & \\
\hline Middlesbrough & 1 & \\
\hline Middlesborough & 1 & \\
\hline New Romney & & 2 \\
\hline Long Eaton & & 2 \\
\hline New York & & 2 \\
\hline Middleton Stoney & & 2 \\
\hline Northbridge & 1 & \\
\hline Southport & 1 & \\
\hline Westfield & 1 & \\
\hline Southampton & 2 & \\
\hline Northampton & 2 & \\
\hline South Haven & & 2 \\
\hline South Bend & & 2 \\
\hline South Berwick & & 2 \\
\hline
\end{tabular}




\begin{tabular}{|c|c|c|}
\hline \multirow{2}{*}{ Nom } & \multicolumn{2}{|c|}{ Constituant accentué } \\
\hline & Toponyme soudé & Toponyme non-soudé \\
\hline Hawthornden & 1 & \\
\hline Bridgeport & 1 & \\
\hline Ivybridge & 1 & \\
\hline Summerfield & 1 & \\
\hline Winterbourne & 1 & \\
\hline Avondale & 1 & \\
\hline Kidderminster & 1 & \\
\hline Water Newton & & 2 \\
\hline Priest Weston & & 2 \\
\hline Astley Abbot & & 2 \\
\hline Saint Albans & & 2 \\
\hline Bishopsgate & 1 & \\
\hline Knightsbridge & 1 & \\
\hline Kingston & 1 & \\
\hline Beaconsfield & 1 & \\
\hline King's Norton & & 2 \\
\hline Bishop's Norton & & 2 \\
\hline Stockton on the Forest & & 4 \\
\hline Stockton on Tees & & 3 \\
\hline Newton-le-Willows & & 3 \\
\hline
\end{tabular}

5.2. Accentuation lexicale par rapport au type fonctionnel des constituants

Le tableau ci-dessous présente quel terme est accentué dans le toponyme, ainsi que le type fonctionnel des constituants.

\begin{tabular}{|c|c|c|}
\hline Nom & Constituant accentué & Type fonctionnel \\
\hline Whitechapel & 1 & $\mathrm{As}+\mathrm{Ag}$ \\
Blackpool & 1 & $\mathrm{As}+\mathrm{Ag}$ \\
Greenfield & 1 & $\mathrm{As}+\mathrm{Ag}$ \\
Whitefriars & 1 & $\mathrm{As}+\mathrm{Ag}$ \\
Blackfriars & 2 & $\mathrm{As}+\mathrm{Ag}$ \\
White House & 1 & $\mathrm{As}+\mathrm{Ag}$ \\
White Hall & 1 & $\mathrm{As}+\mathrm{Ag}$ \\
\hline
\end{tabular}




\begin{tabular}{|c|c|c|}
\hline Nom & Constituant accentué & Type fonctionnel \\
\hline Oldbury & 1 & $\mathrm{As}+\mathrm{Ag}$ \\
\hline Highbridge & 1 & $\mathrm{As}+\mathrm{Ag}$ \\
\hline Newhaven & 1 & $\mathrm{As}+\mathrm{Ag}$ \\
\hline New Haven & 2 & $\mathrm{As}+\mathrm{Ag}$ \\
\hline New Romney & 2 & $\mathrm{As}+\mathrm{Pg}$ \\
\hline New York & 2 & $\mathrm{As}+\mathrm{Pg}$ \\
\hline Great Yarmouth & 2 & $\mathrm{As}+\mathrm{Pg}$ \\
\hline Little Rock & 1 & $\mathrm{As}+\mathrm{Ag}$ \\
\hline Westfield & 1 & $\mathrm{As}+\mathrm{Ag}$ \\
\hline Northbridge & 1 & $\mathrm{As}+\mathrm{Ag}$ \\
\hline Southport & 1 & $\mathrm{As}+\mathrm{Ag}$ \\
\hline Southampton & 2 & $\mathrm{As}+\mathrm{Ag}$ \\
\hline Southend & 2 & $\mathrm{As}+\mathrm{Ag}$ \\
\hline Eastbourne & 1 & $\mathrm{As}+\mathrm{Pg}$ \\
\hline South Haven & 2 & $\mathrm{As}+\mathrm{Ag}$ \\
\hline South Shields & 2 & $\mathrm{As}+\mathrm{Ag}$ \\
\hline South Berwick & 2 & $\mathrm{As}+\mathrm{Pg}$ \\
\hline Bridgewater & 1 & $\mathrm{As}+\mathrm{Ag}$ \\
\hline Bridgeport & 1 & $\mathrm{As}+\mathrm{Ag}$ \\
\hline Ivybridge & 1 & $\mathrm{As}+\mathrm{Ag}$ \\
\hline Avondale & 1 & $\mathrm{Ps}+\mathrm{Ag}$ \\
\hline Market Rasen & 2 & $\mathrm{As}+\mathrm{Pg}$ \\
\hline Chipping Norton & 2 & $\mathrm{Ps}+\mathrm{Pg}$ \\
\hline Lyme Regis & 2 & $\mathrm{As}+\mathrm{Pg}$ \\
\hline Saint Albans & 2 & $\mathrm{As}+\mathrm{Pg}$ \\
\hline Bishopsgate & 1 & $\mathrm{As}+\mathrm{Ag}$ \\
\hline Kingston & 1 & $\mathrm{As}+\mathrm{Ag}$ \\
\hline Kingswood & 1 & $\mathrm{As}+\mathrm{Ag}$ \\
\hline King’s Norton & 2 & $\mathrm{As}+\mathrm{Pg}$ \\
\hline Bishop's Norton & 2 & $\mathrm{As}+\mathrm{Pg}$ \\
\hline King's Cliffe & 2 & $\mathrm{As}+\mathrm{Ag}$ \\
\hline Stockton on the Forest & 4 & $\mathrm{Pg}+$ prep + art $+\mathrm{Ag}$ \\
\hline Stockton on Tees & 3 & $\mathrm{Pg}+$ prep + Ps \\
\hline Norton-le-Willows & 3 & $\mathrm{Pg}+$ prep $+\mathrm{Ag}$ \\
\hline Walton on the Hill & 4 & $\mathrm{Pg}+$ prep + art $+\mathrm{Ag}$ \\
\hline
\end{tabular}

\section{En guise de conclusion}

Nous pouvons dire que, contrairement à ce qui se passe dans d'autres langues, comme en allemand par exemple, l'accentuation des noms de villes anglaises composés 


\section{$L$ 'accentuation des noms de ville en anglais}

de plusieurs termes transparents ou semi-transparents (soudés ou non soudés) n'est pas affectée par le nombre de syllabes que compte le toponyme. L'accentuation de ce type de toponymes suit en général les règles qui s'appliquent en anglais aux noms composés, avec quelques exceptions, et dépend de la nature morphosyntaxique du toponyme considéré. Les toponymes soudés sont en général accentués sur le premier terme, alors que les toponymes non-soudés sont le plus souvent accentués sur le deuxième terme ou à droite pour les noms de plus de deux éléments (voir tableaux ci-dessus). On peut également remarquer que les noms de villes composés d'éléments non soudés sont souvent de type $\mathrm{As}+\mathrm{Pg}$, mais ils peuvent aussi être de type $\mathrm{Ps}+\mathrm{Pg}$, As $+\mathrm{Ag}$, ou intégrer une préposition. Certains noms de ville formés de composés soudés ont également le type de schéma fonctionnel As + Ag. Si le type fonctionnel peut orienter le choix du locuteur lorsqu'il ne sait pas précisément quelle syllabe est accentuée dans un toponyme de ce genre, l'analyse de sa structure (soudée ou non-soudée) semble en fait être un élément plus déterminant encore.

Certains noms de villes américaines suivent également ce modèle, comme nous avons pu le voir avec les quelques exemples cités dans cette étude, mais une étude plus complète mériterait d'être consacrée à ces toponymes.

\section{Bibliographie}

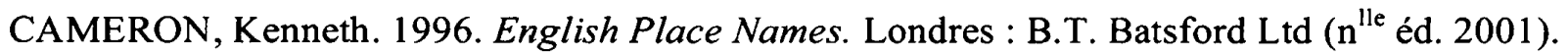

JONES, Daniel. 1997. English Pronouncing Dictionary. Cambridge : Cambridge University Press ( $15^{\mathrm{e}}$ éd. par Peter Roach \& James Hartman).

Merriam-Webster's Geographical Dictionary. 1997. Springfield, Massachusetts, U.S.A. : Merriam-Webster Incorporated.

VAN LANGENDONCK, Willy. 2007. Theory and Typology of Proper Names. Berlin; New York : Mouton de Gruyter. 